\title{
Rheology printing of an ultra-fine conductive Ru-La-O line
}

\author{
Koji Nagahara, ${ }^{\mathrm{a},}$ Daisuke Hirose, ${ }^{\mathrm{a}}$ Jinwang Li, ${ }^{\mathrm{b}}$ Junichi Mihara ${ }^{\mathrm{a}}$ and Tatsuya Shimoda ${ }^{\mathrm{a}, \mathrm{b}}$ \\ ${ }^{\mathrm{a}}$ School of Materials Science, Japan Advanced Institute of Science and Technology (JAIST), 1-1 Asahidai, Nomi, \\ Ishikawa 923-1292, Japan. \\ ${ }^{\mathrm{b}}$ Green Devices Research Center, Japan Advanced Institute of Science and Technology (JAIST), 2-13 Asahidai, \\ Nomi, Ishikawa 923-1211, Japan.
}

\begin{abstract}
We previously developed a method for producing highly conductive $\mathrm{RuO}_{2}$ films using a solution-based process. In this paper, we describe the further development of this material to produce fine patterns for use in nano-sized devices through a newly developed direct imprinting process, named nano-Rheology Printing (n-RP). This process involves the fine patterning of the precursor gel by an imprinting process to generate fine solid patterns while avoiding potential pattern breakage during sintering. The pattern breaking is typically caused by the crystallization nature of an oxide material, which may become more prominent when the size is reduced to less than $100 \mathrm{~nm}$. To solve this problem, we investigated the properties of the Ru-La system and observed that a small addition of La to $\mathrm{Ru}$ greatly improved the viscoelastic property of the gel without any noticeable reduction of conductivity. The $\mathrm{Ru}$-La system also exhibited an amorphous state over a wide composition range, and thus the fine gel patterns produced by the imprinting process were resistant to breakage during sintering. These unique properties of the Ru-La system were thought to result from the molecular crystalline nature of the La gel. Based on the results, the $\mathrm{n}$-RP method could successfully fabricate a fine line of the $\mathrm{Ru}_{0.75}-\mathrm{La}_{0.25} \mathrm{O}$ oxide with a width of $30 \mathrm{~nm}$ and with good conductivity.
\end{abstract}

\section{Introduction}

A great attention has been paid to printing technologies such as inkjet, screen and gravure printings as direct processing methods to fabricate various precise devices. ${ }^{1-5}$ Since the printing method patterns the devices directly from functional inks, the process becomes very simple with maximum efficiency in the use of raw materials. Therefore, the direct methods have advantages over conventional processes such as vacuum deposition and photolithography in terms of production costs and environmental burdens. However, the printing technologies developed until now have serious issues in regards to poor precision and limited pattern shapes; i.e. the smallest pattern size by printing is limited to more than $10 \mu \mathrm{m}$ and the shape of the printed pattern is not well-defined as it tends to have rounded edges with no clear edge and low aspect ratios. ${ }^{6-8}$

In order to solve the problems noted above, we have developed a new printing method named nano-Rheology Printing (n-RP) using an imprinting technology. ${ }^{9}$ This method may be used to fabricate well-defined and clear-edged metal-oxide patterns of sizes $<1 \mu \mathrm{m}$. Whereas conventional nanoimprint lithography (NIL) uses a photoresist process for patterning, ${ }^{10-12}$ the n-RP method can form an oxide gel pattern directly by thermal 
imprinting. In the n-RP process, the development of an oxide gel material that has plasticity under thermal conditions is critical. For this purpose, we have already developed several kinds of oxide materials, including

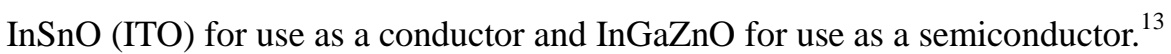

To further develop the n-RP technology, expansion of the material species, enhancement of the processing ability, and development of a nanofabrication technology are necessary. Among these, the development of a nanofabrication technology is crucial because the n-RP process is one of the only technologies that is capable of accommodating requests for nanopatterns $<100 \mathrm{~nm}$, which are necessary in the semiconductor business. When $\mathrm{n}$-RP is applied to the fabrication of fine patterns, however, problems such as technical difficulties in imprinting a fine gel pattern and a phenomenon of spontaneous pattern breaking during sintering may be observed. Therefore, the development of dedicated technologies to form fine patterns is necessary, in which compositional searches, the design of the solution, analysis of the gel structure and its deformation mechanism, and analysis of sintered substances will be the research themes.

We recently succeeded in patterning the RuO electrodes of a thin-film transistor (TFT) by n-RP, where RuO exhibited high conductivity. ${ }^{14,15}$ We attempted to make a fine pattern of RuO by the same method, but only confirmed that the pattern broke during sintering because of its crystallization and grain growth. In this case, the use of an amorphous material may help to avoid the breaking caused by crystallization. One possible amorphous material that also has high conductivity is $\mathrm{RuLaO} .{ }^{16,17}$ In this study, we report that the plastic deformation ability was greatly improved by adding $\mathrm{La}$ to Ru to allow for the formation of a nano-sized gel pattern by $\mathrm{n}-\mathrm{RP}$. The gel pattern was successfully converted to an amorphous $\mathrm{RuLaO}$ structure without breaking during sintering. As a result, we succeeded in the fabrication of a RuLaO narrow line with a width of a few tens of nanometres. We also investigated the structures of solutions, gels, and solids, and the conversion process from solution to solid via gel by thermogravimetry-differential thermal analysis (TG-TDA), lab X-ray diffraction (XRD), Fourier transform infrared spectroscopy (FT-IR), high-energy XRD (HEXRD) using a synchrotron radiation source, mass spectrometry, and thermo-rheological analysis to understand why RuLaO had a high plastic deformation ability during the n-RP process. As RuLaO can be patterned into a semiconductor compatible size by $\mathrm{n}-\mathrm{RP}$, this material is expected to have applications as an electrode material in future electronics.

\section{Experimental}

\section{Solution and gel preparation}

The solutions were prepared by dissolving different amounts of Ruthenium(III) nitrosyl acetate (Ru-NO-ac, $\mathrm{Ru}[\mathrm{NO}]\left[\mathrm{CH}_{3} \mathrm{COO}\right]_{3} ; 99.99 \%$, Alfa Aesar) and Lanthanum(III) acetate hydrate ( $\mathrm{La}-\mathrm{ac}, \mathrm{La}\left[\mathrm{CH}_{3} \mathrm{COO}\right]_{3} \cdot 1.5 \mathrm{H}_{2} \mathrm{O}$; 99.99\%, Kanto Chemical) with monoethanolamine (MEA, $\mathrm{NH}_{2} \mathrm{C}_{2} \mathrm{H}_{4} \mathrm{OH} ; 99.0 \%$, Kanto Chemical) in propionic acid $\left(\mathrm{C}_{2} \mathrm{H}_{5} \mathrm{COOH} ; 99.3 \%\right.$, Kanto Chemical) to form a $0.4 \mathrm{M}$ solution (representing the total concentration of the $\mathrm{Ru}$ and La precursor mixtures) in a sealed glass vial, which was heated for 1 hour on a metal hotplate set at $110{ }^{\circ} \mathrm{C}$ with magnetic stirring set at $1000 \mathrm{rpm}$. The molar ratio of MEA to the total amount of metals was 2:1. Caution: MEA should be added dropwise into propionic acid since heat is generated upon the addition, accompanied by an apparent temperature increase. Five different solutions were prepared by altering the molar percentages of La-ac 
and Ru-NO-ac as follows: Ru100 (100\% Ru, 0\% La), Ru75La25 (75\% Ru, 25\% La), Ru50La50 (50\% Ru, 50\% La), Ru25La75 (25\% Ru, 75\% La), and La100 (0\% Ru, 100\% La).

\section{Thermogravimetry-differential thermal analysis (TG-DTA)}

Information regarding the thermal behaviour (evaporation, decomposition, and crystallisation) and the temperature range for precursors applied for n-RP was obtained by TG-DTA. The measurements were carried out in air using a rising temperature rate of $10^{\circ} \mathrm{C} \mathrm{min}^{-1}$ with an EXSTAR6000 instrument (SII-nanotech, Japan).

\section{Lab X-ray diffraction $(X R D)$ analysis}

The structural difference of the films prepared from the solutions with composition variations was investigated by XRD analysis. Each solution was spin-coated on $\mathrm{a} \mathrm{SiO}_{2} / \mathrm{Si}$ substrate at $1500 \mathrm{rpm}$, followed by heating at $100-$ $400{ }^{\circ} \mathrm{C}$ for $10 \mathrm{~min}$ in air. The resulting films were measured on an X-ray diffractometer (X'Pert PRO MRD Epi, PANalytical).

\section{Resistance and thickness measurements}

Samples for resistance measurements were fabricated by spin-coating each solution onto a $\mathrm{SiO}_{2} / \mathrm{Si}$ substrate at $1500 \mathrm{rpm}$, followed by annealing at $400{ }^{\circ} \mathrm{C}$ for $10 \mathrm{~min}$ in the ambient atmosphere. The four-probe method for low resistance measurements and the double-ring method for high resistance measurements were used. For the thickness measurement, a probe-type step profiler (Alpha-Step 500, KLA-Tencor) was used.

\section{Investigation of the nano-Rheology Printing $(R P)$ properties}

The term nano-Rheology Printing (n-RP) refers to the total process including imprinting followed by post-sintering, while the term Rheology Printing (RP) refers to the imprinting process using gel materials.

In order to investigate the n-RP properties, RP patterning experiments for the gel of each ratio of metals was performed. Fig. 1 shows the process in detail. First, gel films were prepared by spincoating solutions onto $\mathrm{SiO} / 2 \mathrm{Si}$ substrates at $1500 \mathrm{rpm}$, followed by drying in air. The drying temperatures of $150{ }^{\circ} \mathrm{C}, 175{ }^{\circ} \mathrm{C}$, and $200{ }^{\circ} \mathrm{C}$ were chosen based on the TG-DTA results. Second, the demoulding treatment was conducted on the mould and the sample surfaces by using the silane coupling agent HD-1100TH (Daikin Industries). A Si mould with a minimum line width of $70 \mathrm{~nm}$ (NIM-80L Reso, NTT-AT) was used. Third, the samples were imprinted at a pressure of 20 MPa for 5 min by using the nanoimprint apparatus ST-50 (Toshiba Machine). Finally, demoulding was performed at $150{ }^{\circ} \mathrm{C}$. The imprinted samples were divided into two parts: one was as imprinted (as-RPed), and the other was further annealed at $400{ }^{\circ} \mathrm{C}$ for $10 \mathrm{~min}$ in air.

For the n-RP of a nano-sized pattern, the Ru25La75-gel was imprinted by the RP method using a specially designed mould (Elionix Inc., Japan) that had a narrow line pattern connected to large pad patterns at both sides. The other conditions were almost the same as those described above. 


\section{Measurement of viscoelastic properties}

The viscoelastic properties were measured for the Ru100-gel and Ru50La50-gel dried at $175^{\circ} \mathrm{C}$, as these two samples were observed to have different RP patterning abilities. To prepare the measurement samples, solutions were dropped onto glass substrates and then dried at $175^{\circ} \mathrm{C}$ for $1 \mathrm{~h}$ in air. The resulting gels were peeled off the substrates and pressed into pellets (100 $\mathrm{mm}^{2}$ large and $0.7 \mathrm{~mm}$ thick), and then measured on a rheometer E-400 (UBM corporation) at a pressure of $1 \mathrm{MPa}$.

\section{Analysis of patterns by scanning electron microscopy (SEM) and atomic force microscopy (AFM)}

The shapes of the as-RPed patterns and annealed small patterns were investigated by SEM and AFM. The patterns were measured on an S-4100 instrument (Hitachi) for SEM analysis and an SPA-400 system (SII-nanotech, Japan) for AFM analysis. The Dynamic Force Microscopy (DFM) mode was used for the AFM measurements.

\section{Analysis of gel structures by Fourier transform infrared (FT-IR) spectroscopy}

The structures of the gels with organic ligands were analysed by FT-IR spectroscopy. Thin film specimens on $\mathrm{SiO}_{2} / \mathrm{Si}$ substrates were prepared by spin coating and drying for $5 \mathrm{~min}$ at different temperatures. We employed the attenuated total reflection method (SpectrumSpotlight200, Perkinelmer).

\section{High-energy X-ray diffraction (HEXRD) experiments}

The structures of the solutions, gels, and solids were investigated by HEXRD using a synchrotron radiation source at beamline BL04B2 (SPring-8, Japan). The energy of the X-rays was $61.4 \mathrm{keV}$ and the measurement was conducted by the $2 \theta$ scan method across an angular range of $0.3^{\circ}$ to $48^{\circ}$ (corresponding to $\mathrm{Q}=0.2-25 \AA$ - -1 ). The total structure factor $S(Q)$ was calculated from the spectrum of the measured intensity $v s .2 \theta$ (angle). The pair distribution function $G(r)$ and the total correlation function $T(r)$ were calculated by a Fourier transformation of $\underline{S}(Q)$.

\section{Analysis of the solution structures by mass spectrometry}

The structures of the precursor solutions were analysed by the Fourier transform-ion cyclotron resonance-mass spectrometry (FT-ICR-MS) using a Solarix-JA instrument (Bruker Daltonics) in the positive ion mode. The solutions for analysis were diluted with methanol by a factor of 1000 . To prevent destruction of the structures in solutions, cold spray ionization (CSI) with liquid nitrogen cooling was employed.

\section{Conversion from solutions to solids}

The specific details of how a solution converts to a solid through the gel state are required in order to perform the n-RP process. In addition, the structure of a solid is highly influential for fabricating a pattern out of the solids, especially a precise pattern using the n-RP method. The conversion process from solutions to solids, the structures of solids, and the electrical properties of films with five different RuLa ratios were investigated. Moreover, the temperature range of the precursors in a gel state was identified to determine the appropriate RP conditions. 


\subsection{Thermogravimetric analysis results}

The results of TG and DTA are shown in Figs. 2 and 3, respectively. The weight loss and endothermic reaction due to the evaporation of solvent was observed from room temperature to more than $100{ }^{\circ} \mathrm{C}$. Since the endothermic reaction ended at approximately $150{ }^{\circ} \mathrm{C}$ according to the DTA results, the evaporation of solvents was also judged to have ended at this temperature. At higher temperatures, a weight reduction with exothermal reaction was observed for all liquids between approximately $260{ }^{\circ} \mathrm{C}$ and $400{ }^{\circ} \mathrm{C}$. This indicated the occurrence of a solidification process for each gel due to oxidization. Based on these results, the gel states of the investigated specimens were observed in the range between $150{ }^{\circ} \mathrm{C}$ and $250{ }^{\circ} \mathrm{C}$.

As thermal imprinting of the gel is the main part of the n-RP process, a detailed understanding of the drying behaviour of the gel is crucial to ensure that the n-RP process is successful. In Table 1, the residual weight of each gel at each temperature was tabulated by taking the weights of the room temperature solutions as 100 . The residual weight of the gel was observed to increase as the ratio of La increased, which indicated that the increased amount of La caused the increase in the residual organics in the gel. In Table 2, the weight ratios of the gels are shown by taking the weight of the $150{ }^{\circ} \mathrm{C}$ gels as 100 . The corresponding magnified figure of TG from $120{ }^{\circ} \mathrm{C}$ to $250{ }^{\circ} \mathrm{C}$ is inserted in Fig. 2. Based on these results, a greater weight reduction was observed when the La ratio became larger. This result was attributed to that larger amounts of the residual organic ingredient maintained by the La compound caused the larger discharge of organic substances upon drying and solidification.

Next, we tried to clarify the difference in weight reductions among the different compositions. For this purpose, the differential TG curves (DTG) shown in Fig.4 were derived from the TG curve. Based on the results, the La100-gel displayed a weight change due to evaporation up to $150^{\circ} \mathrm{C}$, followed by a relatively large weight change up to $250{ }^{\circ} \mathrm{C}$ before solidification occurred that was accompanied by several weight reductions from $300{ }^{\circ} \mathrm{C}$ to $400{ }^{\circ} \mathrm{C}$. In contrast, the Ru100-gel lost its weight by evaporation up to $150{ }^{\circ} \mathrm{C}$ and was followed by a relatively smaller weight change at higher temperatures compared to the La100-gel. The Ru100-gel showed a small characteristic peak at $200^{\circ} \mathrm{C}$. This peak corresponded to the exothermal peak in the DTA results (Fig. 3), and thus some type of oxidation process was thought to have occurred at this temperature. At higher temperatures, a large weight reduction occurred from $250^{\circ} \mathrm{C}$ to $300^{\circ} \mathrm{C}$ due to solidification of the gel. These results clearly showed that there was a large difference in the thermal behaviours between the La100-gel and Ru100-gel.

For the DTG curves of the RuLa compositions, the curve for Ru25La75 was similar to that of La100, and the curves of both Ru50La50 and Ru75La25 showed the same tendencies as that of the Ru100-gel. This meant that each gel in the RuLa system showed the same tendency of either La100 or Ru100, resulting in no observation of an intermediate state. Therefore, the structure of RuLa-gel could be a mixture of La100-gel and Ru100-gel, and this structure could govern the n-RP behaviour.

\subsection{XRD analysis for characterization of crystallinity}

The obtained XRD patterns are presented in Figs. 5 (a) - (e). The XRD patterns of the annealed Ru100 and La100 samples showed diffraction lines representative of $\mathrm{RuO} 2$ and La2O3 crystals, respectively, while those of the samples containing both $\mathrm{La}$ and $\mathrm{Ru}$ displayed no diffraction lines of these crystals. Thus, the solid films containing both La and Ru were amorphous. To clarify this, HEXRD measurements were performed for Ru100, 
La100, and Ru50La50-oxide. The Ru50La50-oxide that was annealed up to $650{ }^{\circ} \mathrm{C}$ displayed no crystalline peaks in the HEXRD pattern (Fig. 5(f)), indicating that it was completely amorphous without local ordering. For the La100 samples, diffraction lines of crystals were observed (Fig. 5(e)) after drying at $100-200{ }^{\circ} \mathrm{C}$, indicating that the La100-gel crystallized in this temperature range. These diffraction lines vanished after heating at $\geq 250{ }^{\circ} \mathrm{C}$. The results of the TG-DTA analysis indicated that the La100-gel contained apparently more organic components than other gels at $150-200{ }^{\circ} \mathrm{C}$, suggesting that the $\mathrm{LaO}$ precursor had stable organic ligands upon the conversion from solution to gel. Accordingly, we considered that the crystals in the La100-gel formed at low temperatures (100$200{ }^{\circ} \mathrm{C}$ ) had organics in their structure, indicating that they were molecular crystals. Because the diffraction lines of these molecular crystals were not observed after heating at $250^{\circ} \mathrm{C}$, these crystals disappeared at this temperature. This result was correlated to the decreased weight loss in the DTG data when the temperature reached $250{ }^{\circ} \mathrm{C}$. The Ru25La75-gel displayed no diffraction lines of molecular crystals, indicating that the addition of Ru changed the gel from a structure of molecular crystals to an amorphous state. Nevertheless, the TG-DTA data of the Ru25La75-solution showed the same thermal behaviour as in those of the La100-solution, which suggested that although the Ru25La75-gel was XRD-amorphous, it contained the basic structure in molecular crystals of the La100-gel. In other words, with the addition of Ru, the long-range ordered structure of molecular crystals was destroyed while the short-range (range of the molecular size) features was maintained.

To summarize the XRD results, for La100, the addition of Ru led to an amorphous gel at low drying temperatures, while for Ru100, the addition of La led to an amorphous solid after annealing. In other words, by mixing $\mathrm{La}$ and $\mathrm{Ru}$ in the RuLa system, amorphous structures could be obtained for both the gel and the annealed solid. This fact is highly important for the fabrication of nanometre-sized lines by $n-R P$. In the n-RP patterning of such lines, lines of a gel are first prepared by thermal imprinting, after which the lines must be converted to a solid by thermal annealing while maintaining the shapes of the lines. During imprinting, a gel in the amorphous state is preferred, while during thermal annealing, a resultant solid in the amorphous state is preferred. In a crystalline material, the formation of particles of crystals would destroy the shape of a line pattern. Accordingly, by mixing La and $\mathrm{Ru}$, both the structures of the gel and the solid that are suitable for n-RP patterning have been realized.

\subsection{Resistivity of RuLa-oxide film}

The resistivities of RuLa-oxide at various RuLa ratios are shown in Fig.6. The Ru100-oxide had a low resistivity of $1.1 \times 10^{-3} \mathrm{ohm} \cdot \mathrm{cm}$, and the addition of La to Ru was observed to cause an increase in resistivity though $25 \%$ of La addition resulted in only a slightly increased resistivity. With the addition of more than $50 \% \mathrm{La}$, the resistivity increased exponentially and with more than $75 \% \mathrm{La}$, the resistivity became close to that of an insulator. In our previous study, the resistivity of Ru100-oxide was $8.0 \times 10^{-4} \mathrm{ohm} \cdot \mathrm{cm}$, which was slightly lower than the result observed in this study. This was attributed to the difference of annealing atmospheres between this study and the previous one. 


\section{Properties of nano-Rheology Printing}

\subsection{Direct observation of patterns}

The optical images of the resultant patterns at each ratio of metals and at each drying temperature are shown in Fig.7. The left side of the boundary line (dashed) shows the as-RPed gel patterns, and the right side shows the patterns after solidification by annealing. For the gels dried at $150{ }^{\circ} \mathrm{C}$, clear patterns were observed in all samples. For those dried at $175{ }^{\circ} \mathrm{C}$, only the Ru100-gel displayed an unclear pattern while the other samples presented clear patterns. Therefore, the RP property was dramatically improved by the addition of the La component. For gels dried at $200{ }^{\circ} \mathrm{C}$, a relatively clear pattern was only observed in the La100-gel, while vague patterns were identified in RuLa-gel and no pattern in Ru100-gel. According to the TG-DTA results, the amount of organic substance in the gel increased with the increasing addition of La. Therefore, the addition of the La component was considered to have kept organic substances in the gels and improved the RP property, even at an increased drying temperature.

After annealing, all the patterns became unclear because the organic substances in the patterns decomposed and the volume shrank. For the Ru100-gel dried at $175^{\circ} \mathrm{C}$, the as-RPed patterns were unclear and the annealed patterns vanished. For the La100-gel, the as-RPed patterns were clear, but the annealed patterns became more unclear than the patterns containing both $\mathrm{La}$ and $\mathrm{Ru}$. This was because the La100-gel experienced the largest volume shrinkage among the samples, which was consistent with its large weight loss observed by TG-DTA (Table 2).

\subsection{Viscoelastic properties of gels}

Figure 8 shows the measured viscoelastic properties. The Ru100-gel displayed a small $\tan \delta$ that maintained stability against temperature variations (Fig. 8(a)). The Ru50La50-gel had tan $\delta$ values that increased from $100{ }^{\circ} \mathrm{C}$ and peaked at approximately $170{ }^{\circ} \mathrm{C}$, which indicated that the gel was more easily softened with the addition of the La component. These results also explained why the gels containing both Ru and La could be imprinted into clear patterns (Fig. 7). Together with the other analyses described above, the results suggested that the addition of La caused more organic components to be retained in the gel, leading to easier softening and higher RP ability.

\subsection{SEM observation of patterns}

The SEM images are shown in Fig. 9. For the La100-gel, an AFM image was also presented because the SEM image was unclear. The drying temperature was $150^{\circ} \mathrm{C}$ for all samples. The as-RPed Ru100-gel and Ru50La50-gel produced clear line and space patterns with a line width of $70 \mathrm{~nm}$. In contrast, the as-RPed La100-gel displayed broken and uneven patterns. This was attributed to the formation of molecular crystals in the La100-gel as observed by the XRD results, which would lead to lower deformation ability. For the annealed Ru100-oxide patterns, the patterned lines were seriously broken and particles of $20-50 \mathrm{~nm}$ in size formed arrays of lines. This was attributed to the crystallization of the Ru100-oxide, during which the lines were broken due to the stress arising from both volume shrinkage and nucleation-growth of crystals. In contrast, the Ru50La50-oxide was amorphous and thus line breaking arising from crystallization did not occur. The La100-gel was not well patterned in imprinting, and the patterns were completely destroyed after annealing. The volume shrinkage of La100 was larger than the other samples, which facilitated the destruction of patterns during annealing. Therefore, RuLa materials containing both 
$\mathrm{La}$ and $\mathrm{Ru}$ were considered to be suitable for the fabrication of small patterns by $\mathrm{n}-\mathrm{RP}$, in contrast to La100 that formed molecular crystals with low imprinting ability, and Ru100 that formed crystals of dozens of nanometres during annealing that resulted in line breaking.

Based on these results, experiments to fabricate conductive small patterns were performed. The Ru75La25 composition was selected, since it displayed conductivity comparable to that of Ru100. The solution was spin-coated onto a $\mathrm{SiO}_{2} / \mathrm{Si}$ substrate followed by drying at $100{ }^{\circ} \mathrm{C}$. Then, $\mathrm{RP}$ was conducted at $150{ }^{\circ} \mathrm{C}$ under 20 $\mathrm{MPa}$. The resulting pattern was annealed at $400{ }^{\circ} \mathrm{C}$ for $10 \mathrm{~min}$ in air. A mould prepared by Elionix Inc. was used, and had a line pattern of $30 \mathrm{~nm}$ in width that was connected to a large pad pattern for probing (for electrical measurement). Figure 10 shows the AFM image of the RuLaO pattern. A clear line of $30 \mathrm{~nm}$ in width was obtained. The conductivity of the line was confirmed by probing on the two pads after Ar plasma etching was performed to remove the residual film. As a result, we succeeded in printing a conductive $\mathrm{RuLaO}$ line of $30 \mathrm{~nm}$ in width.

\section{Analysis of gels and solutions}

\subsection{FT-IR Analysis}

The FT-IR spectra are shown in Fig. 11(a). Many peaks were located within the range from 1000 to $2000 \mathrm{~cm}-1$. These peaks resulted from organic molecules of the solvent, ligands of the solutes, and the additive substance. An abundance of organic molecules were observed to have remained in all gels. The spectra for the La100-gel and Ru100-gel were compared in the range from 750 to $1200 \mathrm{~cm}^{-1}$ (Fig. 11(b)). Peak 1 at $890 \mathrm{~cm}^{-1}$ and peak 3 at 1070 $\mathrm{cm}^{-1}$ were observed in the La100-gel, while peak 2 at $1060 \mathrm{~cm}^{-1}$ was observed in the Ru100-gel. In the RuLa-gel, all three of these peaks were observed, suggesting that the RuLa-gel consisted of the basic structures of both the La100-gel and Ru-100-gel.

\subsection{HEXRD analysis}

To investigate the structures of gels in detail, high-energy $\mathrm{x}$-ray diffraction (HEXRD) with pair distribution function analysis was performed. ${ }^{18}$ The resulting $S(Q)$ and $T(r)$ curves are shown in Figs. 12 and 13, respectively. In the $S(Q)$ curve of La100-gel, sharp peaks indicating a well ordered structure (crystals) and broad peaks (pointed as S1 and S2) indicating a less ordered structure of the La100-gel were observed. These peaks were consistent with the previously obtained XRD patterns. The Ru50La50-gel and Ru75La25-gel displayed S1 and S2 peaks corresponding to the less ordered structure of the La100-gel, indicating the presence of the basic structure of the La100-gel in these RuLa mixed gels. In the $T(r)$, six peaks were observed in the gel samples. Peak T2 was observed in the Ru100-gel, and peaks T1, T3, T4, T5, and T6 were observed in all samples. Peaks T3 and T6 of the La100-gel were larger than those of the Ru100-gel, and peaks T3 and T6 were considered to correspond to La-O and La-La, respectively. To further clarify the peak assignments, the $T(r)$ of La100-oxide and Ru100-oxide were also investigated (Fig. 13(b)). The observed peak position for La-La in the La100-gel was at a distance of $\sim 4.2 \AA$, which was much longer than that observed in the La oxide $(\sim 3.8 \AA)$ and consistent with the MS observations that suggested that the La100-solution had no La-O-La core structures. This result provided further evidence that the La100-gel had a molecular crystalline nature. We considered that the existence of peaks T3 and T6 in the Ru50La50-gel and Ru75La25-gel resulted from the addition of the structure in the La100-gel. From $S(Q)$ and $T(r)$, 
we observed that the RuLa gels had the structures of both the La100-gel and Ru100-gel. The Ru75La25-gel, which was prepared by the addition of La, was observed to have the substantial structure found in the La100-gel. This structure retained organic molecules and improved the RP property.

\subsection{Mass spectrometric analysis}

Figs. 14 (a) - (e) show the MS spectra obtained from analysis of the five different solutions listed in Table 1. For the Ru100-solution (Fig. 14 (a)), an organic ligand-coordinated structure with 1 or 2 Ru atoms was identified, based on a comparison of the calculated and measured isotopic MS spectra. For the La100-solution (Fig. 14 (e)), a regular $\mathrm{m} / \mathrm{z}$ interval of 357.993 that was attributed to $\mathrm{La}[\mathrm{EtCOO}] 3$ was confirmed. The line at a low $\mathrm{m} / \mathrm{z}$ of 407.069 was identified to be a metal-organic structure with $1 \mathrm{La}$ atom. Therefore, in the La100-solution, La formed a basic structure with $1 \mathrm{La}$ atom coordinated by organic ligands. This basic structure further combined each other to form larger ones, upon drying without a condensation reaction that would have released ligands and thus without the formation of an oxide La-O-La core. In the spectra of the RuLa solutions containing both La and Ru (Fig. 14 $(b, c, d))$, the MS lines were same as observed in the spectra for Ru100 and La100, and no new lines were observed. Therefore, the La-structure and Ru-structure in the RuLa solutions did not react and therefore existed only in a mechanically mixed state.

\section{Discussion of the n-RP mechanism}

Based on the results of the viscoelastic analysis for gels dried at $175^{\circ} \mathrm{C}$, the tan $\delta$ of the Ru100-gel did not change, while that of the Ru50La50-gel reached a peak value at approximately $170{ }^{\circ} \mathrm{C}$. This was indicative of the easiness of softening achieved by the addition of La and corresponded to the observed RP properties. Based on the TG-DTA results, the RP patterning ability clearly increased with increasing organic content in the gel. Therefore, the addition of La was correlated to the retention of organic components in the gel, which resulted in softening of the gel and in improved RP patterning ability.

The reason why the La compound led to better retention of organic components was attributed to its ability to form molecular crystals at $100-200{ }^{\circ} \mathrm{C}$. The decomposition temperature of the organic components in the $\mathrm{La}$ compound increased through the formation of such crystals. Upon mixing with the Ru compound, the La compound was considered to work in the same way to improve the ability to retain organic components. From the HEXRD results, the structure of the La100-gel appeared to partially exist in the RuLa gels, which dramatically improved the RP properties.

According to the results of the MS analysis, the Ru compound and La compound existed separately in the RuLa solutions, where they were assumed to be only mixed in a mechanical way. According to the FT-IR data, this mechanically mixed state was maintained in the gels, indicating that $\mathrm{La}$ and $\mathrm{Ru}$ were not in a bonded structure in the RuLa gels but rather the basic structures of the $\mathrm{La}$ and $\mathrm{Ru}$ gels were only mixed. Because of the ability to form molecular crystals, the structure of the La gel was considered to have the ability to maintain organic components even at high temperatures. A high content of organic components resulted in an improved RP patterning ability. Therefore, the RP patterning ability was considerably improved through the addition of the La compound to the Ru solution. 
In the fabrication of small patterns, crystallization both in the gel and during annealing is highly detrimental. The La100-gel crystallized at the temperature of imprinting and could not form clear patterns. On the other hand, while the Ru100-gel gel was able to be patterned, the small patterns were broken upon crystallization during annealing. We succeeded in preventing crystallization both in gels and in annealed oxides through the mixing of La and $\mathrm{Ru}$ compounds, and achieved an amorphous state throughout the process.

Concerning the electrical properties, since the resistivity increased with the addition of $\mathrm{La}$, a small amount of La addition was necessary. Fortunately, for the improvement of the n-RP properties, only a low content of La was necessary. In the present study, the optimum composition was determined to be $25 \% \mathrm{La}$ and $75 \% \mathrm{Ru}$, and the Ru75La25-oxide displayed similar resistivity to the Ru100-oxide. Through the addition of La, the crystallization during annealing was prevented and an amorphous phase was obtained; consequently, the breaking of patterns due to crystallization was avoided and small patterns of $30 \mathrm{~nm}$ in width were achieved. Both the high patterning ability of the La gel and the high conductivity of Ru were maintained through mixing La and Ru compounds.

\section{Conclusions}

A small-sized RuLaO pattern was successfully formed by the n-RP method. This result could not be achieved by sole use of $\mathrm{La}$ or Ru solution, but became possible by the mixture of $\mathrm{La}$ and Ru solutions. Based on the mass spectrum data, the developed RuLa solution was a solution in which the La and Ru solvents were independently dispersed. Therefore, a gel was thought to have the same kind of structure, consisting of a mechanical mixture of La clusters and Ru clusters. The gel state was found between $150^{\circ} \mathrm{C}$ and $250{ }^{\circ} \mathrm{C}$ in the RuLa system. One of the most impressive features of these gels was that the La100-gel displayed a strong molecular crystallinity in the temperature range from 100 to $200{ }^{\circ} \mathrm{C}$, which was made clear by the XRD analysis. This results meant that a La cluster in a solution could be stabilized by the cohesion energy derived from the molecular crystallinity, which in turn increased the desorption temperature of organic elements from a gel.

The resulting gel was thermally imprinted in the RP process. At a temperature of $100{ }^{\circ} \mathrm{C}$, the five compounds investigated were all imprinted relatively well, with the exception of the La100-gel. In this gel, the shapes of relatively small patterns were seriously degraded. During imprinting at $175{ }^{\circ} \mathrm{C}$, the imprinting ability of Ru100-gel was greatly decreased while the other four gels displayed clear imprinted patterns. This result indicated that the addition of La element to Ru was able to improve the imprinting property. This was also confirmed by the viscoelastic measurements, where the tan $\delta$ of the Ru50La50-gel was greatly enhanced compared with that of the Ru100-gel. When the temperature was elevated up to $200{ }^{\circ} \mathrm{C}$, small deformations were only observed in the La100-gel, while the other gels displayed almost no deformation.

After sintering the gel, the structure and conductivity of the solid film were investigated, and the results confirmed that both the films of $\mathrm{LaO}$ and $\mathrm{RuO}$ were crystals, while those of $\mathrm{RuLaO}$ were amorphous. Higher resistivity was also observed when increasing amounts of La element were added, though the decrease of conductivity was relatively small with up to $25 \%$ of La addition.

Based on the above results, we succeeded in using the n-RP method to fabricate a fine line pattern of the Ru75La25-gel that was able to be sintered without any breakage. The resultant line had a width of $30 \mathrm{~nm}$ with good conductivity. This fine patterning was attributed to the following two reasons: the retention of organic elements at 
higher temperatures in the La containing gel due to its molecular crystallinity nature, and the amorphous structure of the RuLa solid that prevented it from spontaneously breaking during sintering.

\section{Acknowledgements}

This work was greatly supported by the members, who belong to JST-ERATO Shimoda Nano-Liquid Process Project, and those of Green Devices Research Center, Japan Advanced Institute of Science and Technology (JAIST). The support of Professor Masayuki Yamaguchi of School of Materials Science, JAIST, for the experiments of viscoelastic properties is appreciated. The high-energy X-ray diffraction experiments were performed using a synchrotron source at SPring- 8 with the approval of the Japan Synchrotron Radiation Research Institute (JASRI) as "Structural analysis of solution-processed new p-type amorphous oxides by using high-energy X-ray diffraction" (Proposal No. 2012B1750/BL No. BL04B2) and "Analysis of the structures of RuLaO solutions and solids by using high-energy X-ray diffraction” (Proposal No. 2015A1875/BL No. BL04B2).

\section{References}

1. D. H. Lee, S.Y. Han, G. S. Herman and C. H. Chang, Inkjet printed high-mobility indium zinc tin oxide thin film transistors, J. Mater. Chem., 2009, 19, 3135-3137.

2. N. M. Kin, B. J. Kim, Y. Ko, J. H. Cho and S. T. Chang, Surface Energy Engineered, High-Resolution Micropatterning of Solution-Processed Reduced Graphene Oxide Thin Films, Adv. Mater., 2013, 25, 894-898.

3. H. Kang, R. Kitsomboonloha, J. Jang and V. Subramanian, High-Performance Printed Transistors Realized Using Femtoliter Gravure-Printed Sub-10 $\mu \mathrm{m}$ Metallic Nanoparticle Patterns and Highly Uniform Polymer Dielectric and Semiconductor Layers, Adv. Mater., 2012, 24, 3065-3069.

4. J. Birnstock, J. Blassing, A. Hunze M. Scheffel, M. Stobel, K. Heuser, G. Wittmann, J. Worle and A. Winnacker, Screen-printed passive matrix displays based on light-emitting polymers, Appl. Phys. Lett., 2001, 78, 3905-3907.

5. K. Fukada, Y. Maeda, X. Y. Liu, A. Matoba, S. Takagi, S. Inoue and T. Shimoda, Fabrication of an All-Screen Printed Oxide Semiconductor Thin Film Transistor Active-Matrix Backplane, SID Int. Symp. Symp. Dig. Tec., 2015, 46, 490-493.

6. H. Sirringhausm T. Kawase, R. H. Friend, T. Shimoda, M. Inbasekaran, W. Wu, E. P. Woo, High-Resolution Inkjet Printing of All-Polymer Transistor Circuits, Science, 2000, 290, 2123-2126.

7. Y. Aleeva and B. Pignataro, Recent advances in upscalable wet methods and ink formulations for printed electronics, J. Mater. Chem. C, 2014, 2, 6436-6453.

8. M. Kawasaki, S. Imazeki, M. Ando, Y. Sekiguchi, S. Hirota, S. Uemura and T. Kamata, High-resolution full-color LCD driven by OTFTs using novel passivation film, IEEE Trans. Electron Dev., 2006, 53, 435-441.

9. T. Kaneda, D. Hirose, T. Miyasako, P. T. Tue, Y. Murakami, S. Kohara, J. Li, T. Mitani, E. Tokumitsu and T. Shimoda, Rheology printing for metal-oxide patterns and devices, J. Mater. Chem. C, 2014, 2, 40-49.

10. S. Y. Chou, P. R. Krauss and P. J. Renstrom, Imprint of sub - $25 \mathrm{~nm}$ vias and trenches in polymers, Appl. Phys. Lett., 1995, 67, 3114-3116. 
11. S. Y. Chou, P. R. Krauss and P. J. Renstrom, Imprint Lithography with 25-Nanometer Resolution, J. Vac. Sci. Technol. B, 1996, 14, 4129-4133.

12. K. Nagahara, B. N. Q. Trinh, E. Tokumitsu, S. Inoue and T. Shimoda, Fabrication of 120-nm-channel-length ferroelectric-gate thin-film transistor by nanoimprint lithography, Jpn. J. Appl. Phys., 2014, 53, 02BC14-1-02BC14-4.

13. D. Hirose and T. Shimoda, Evaluating the state of indium-tin oxide gels via estimation of their cohesive energy, Jpn. J. Appl. Phys., 2014, 53, 02BC01-1-02BC01-7.

14. Y. Murakami, J. Li, D. Hirose, S. Kohara and T. Shimoda, Solution processing of highly conductive ruthenium and ruthenium oxide thin films from ruthenium-amine complexes, J. Mater. Chem. C, 2015, 3, 4490-4499.

15. Y. Murakami, J. Li and T. Shimoda, Highly conductive ruthenium oxide thin films by a low-temperature solution process and green laser annealing, Mater. Lett., 2015, 152, 121-124.

16. P. Khalifah, R. Osborn, Q. Huang, H. W. Zandbergen, R. Jin, Y. Liu, D. Mandrus and R. J. Cava, Orbital Ordering Transition in $\mathrm{La}_{4} \mathrm{Ru}_{2} \mathrm{O}_{10}$, Science, 2002, 297, 2237-2240.

17. J. Li, T. Kaneda, E. Tokumitsu, M. Koyano, T. Mitani and T. Shimoda, P-type conductive amorphous oxides of transition metals from solution processing, Appl. Phys. Lett., 2012, 101, 052102-1-052102-5.

18. T. Proffen, S. J. L. Billinge, T. Egami and D. Louca, Structural analysis of complex materials using the atomic pair distribution function — a practical guide, Zeitschrift für Kristallographie, 2003, 218, 132-143. 
Table 1 The residual weight of each gel at the specific temperatures obtained by taking the weights of the solutions at room temperature as 100 .

\begin{tabular}{lccccc}
\hline & RT. & $150^{\circ} \mathrm{C}$ & $200^{\circ} \mathrm{C}$ & $250^{\circ} \mathrm{C}$ & $500^{\circ} \mathrm{C}$ \\
\hline Ru100 & 100 & 15.8 & 13.7 & 12.2 & 5.7 \\
Ru75La25 & 100 & 18.3 & 15.2 & 13.9 & 6.5 \\
Ru50La50 & 100 & 20.5 & 16.6 & 15.1 & 7.2 \\
Ru25La75 & 100 & 25.1 & 18.7 & 15.8 & 6.6 \\
La100 & 100 & 26.4 & 19.5 & 14.5 & 6.9 \\
\hline
\end{tabular}

Table 2 The residual weight of each gel at the specific temperatures obtained by taking the weight of the $150^{\circ} \mathrm{C}$-dried gels as 100 .

\begin{tabular}{lccccc}
\hline & RT. & $150^{\circ} \mathrm{C}$ & $200^{\circ} \mathrm{C}$ & $250^{\circ} \mathrm{C}$ & $500^{\circ} \mathrm{C}$ \\
\hline Ru100 & - & 100 & 86.7 & 77.2 & 36.1 \\
Ru75La25 & - & 100 & 83.1 & 76.0 & 35.5 \\
Ru50La50 & - & 100 & 81.0 & 73.7 & 35.1 \\
Ru25La75 & - & 100 & 74.5 & 62.9 & 26.3 \\
La100 & - & 100 & 73.9 & 54.9 & 26.1 \\
\hline
\end{tabular}

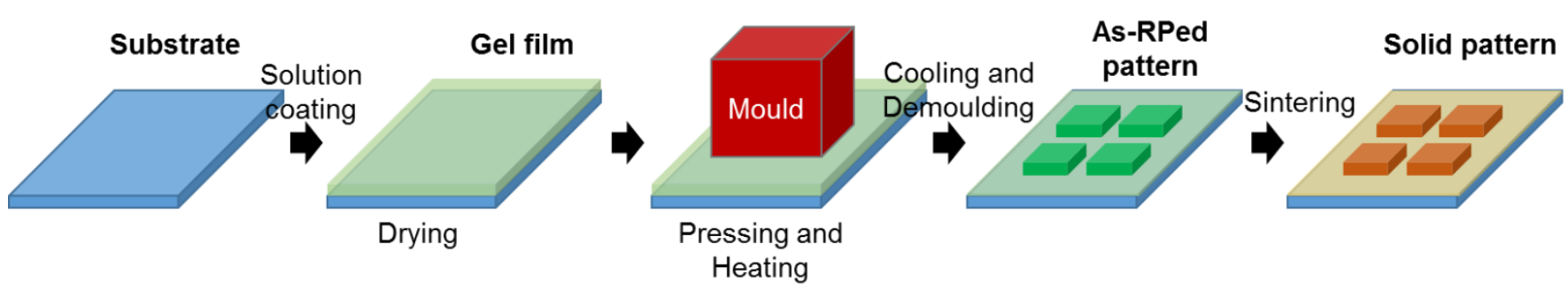

Fig. 1 


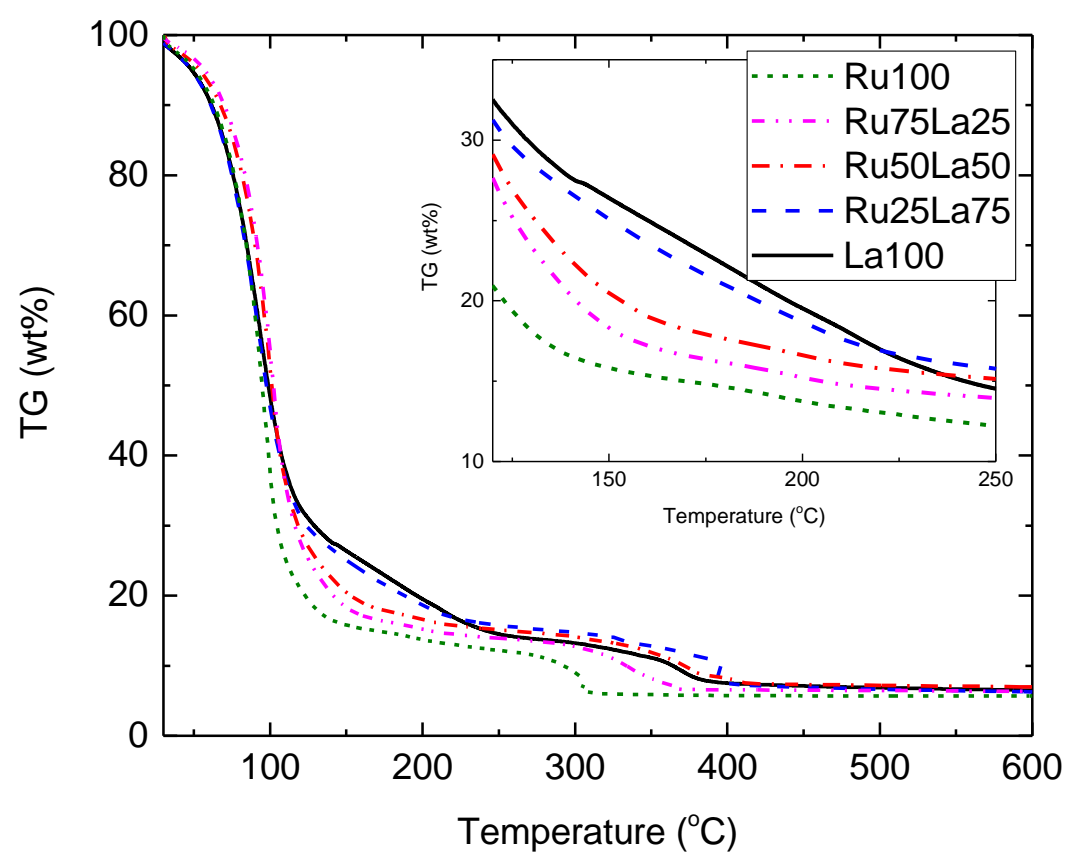

Fig. 2

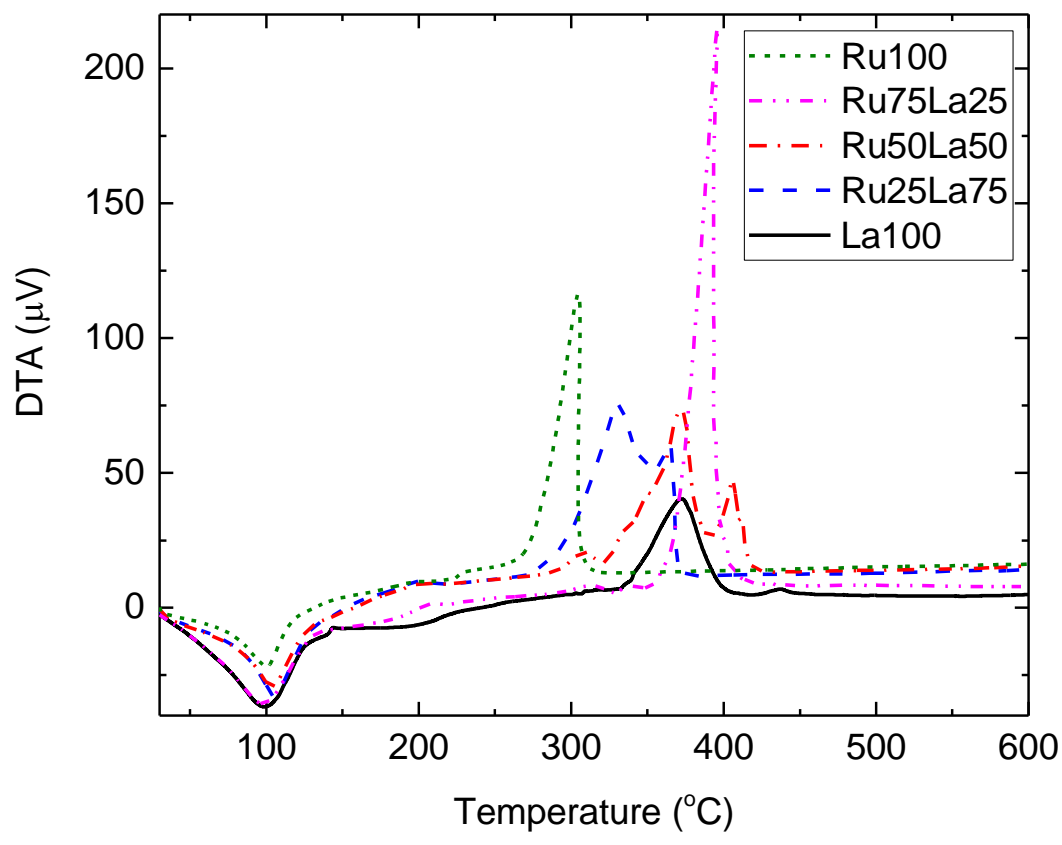

Fig. 3 
1

2

3

4

5

6

7

8

10

11

12

13

14

15

16

17

18

19

20

21

22

23

24

25

26

27

28

29

30

31

32

33

34

35

36

37

38

39

40

41

42

43

44

45

46

47

48

49

50

51

52

53

54

55

56

57

58

59

60

61

62

63

64

65

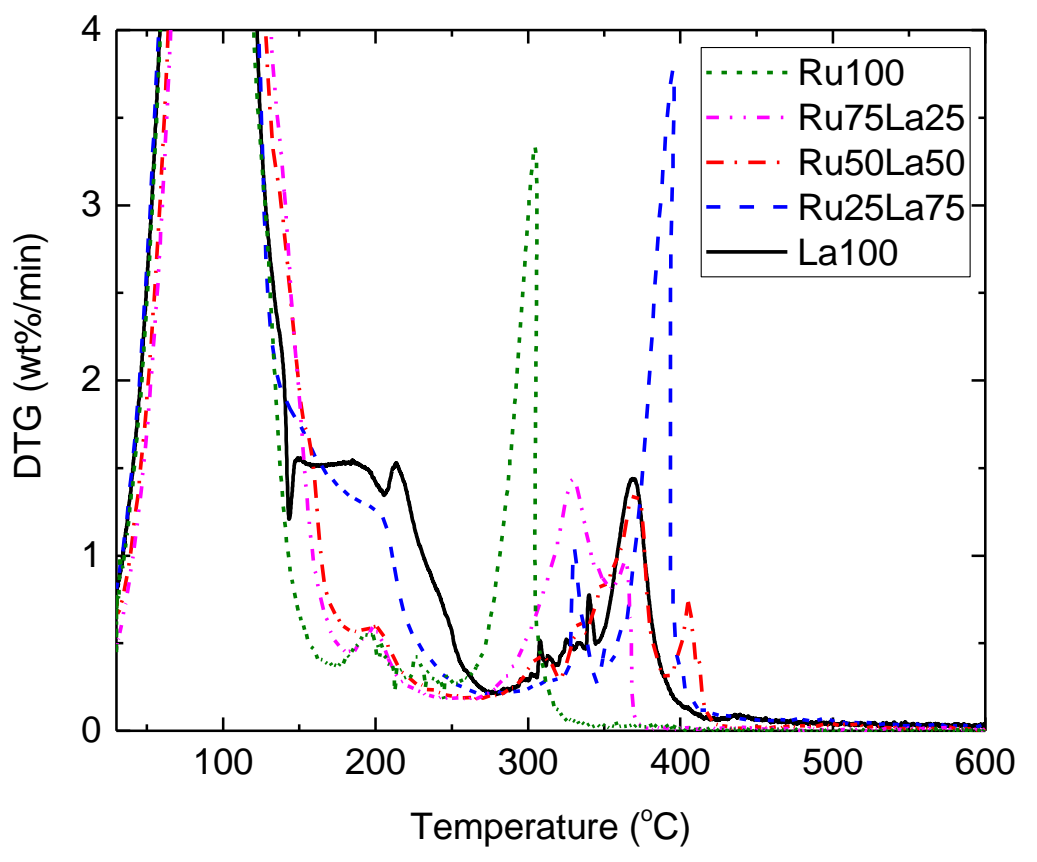

Fig. 4 
(a)

(b)

(a) Ru100

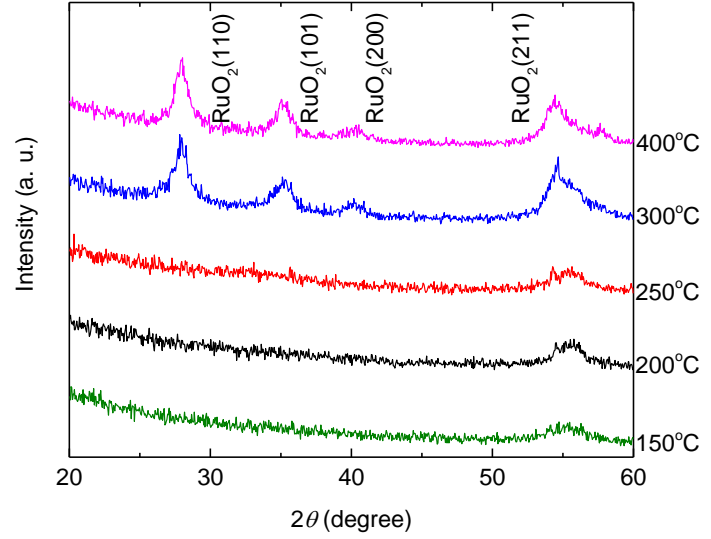

(c)

(c) Ru50La50

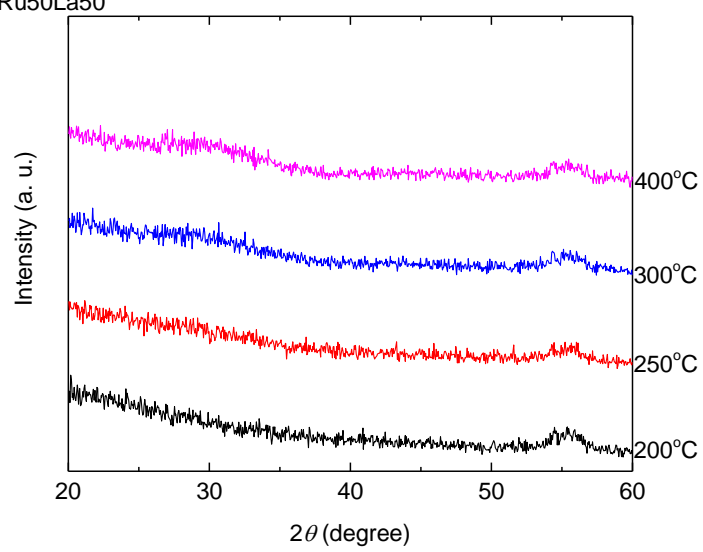

(e)

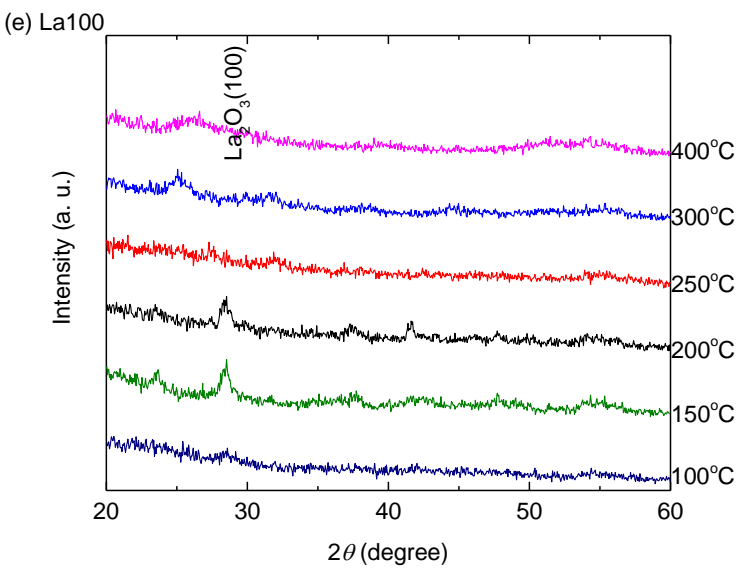

Fig. 5 (b) Ru75La25

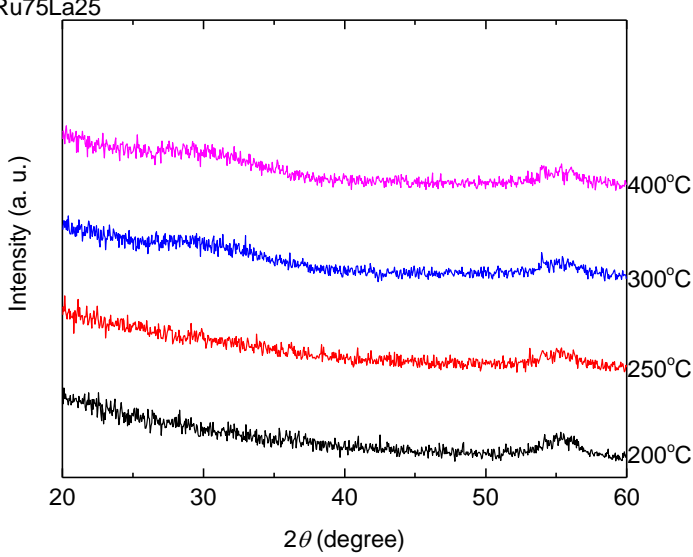

(d) Ru25La75
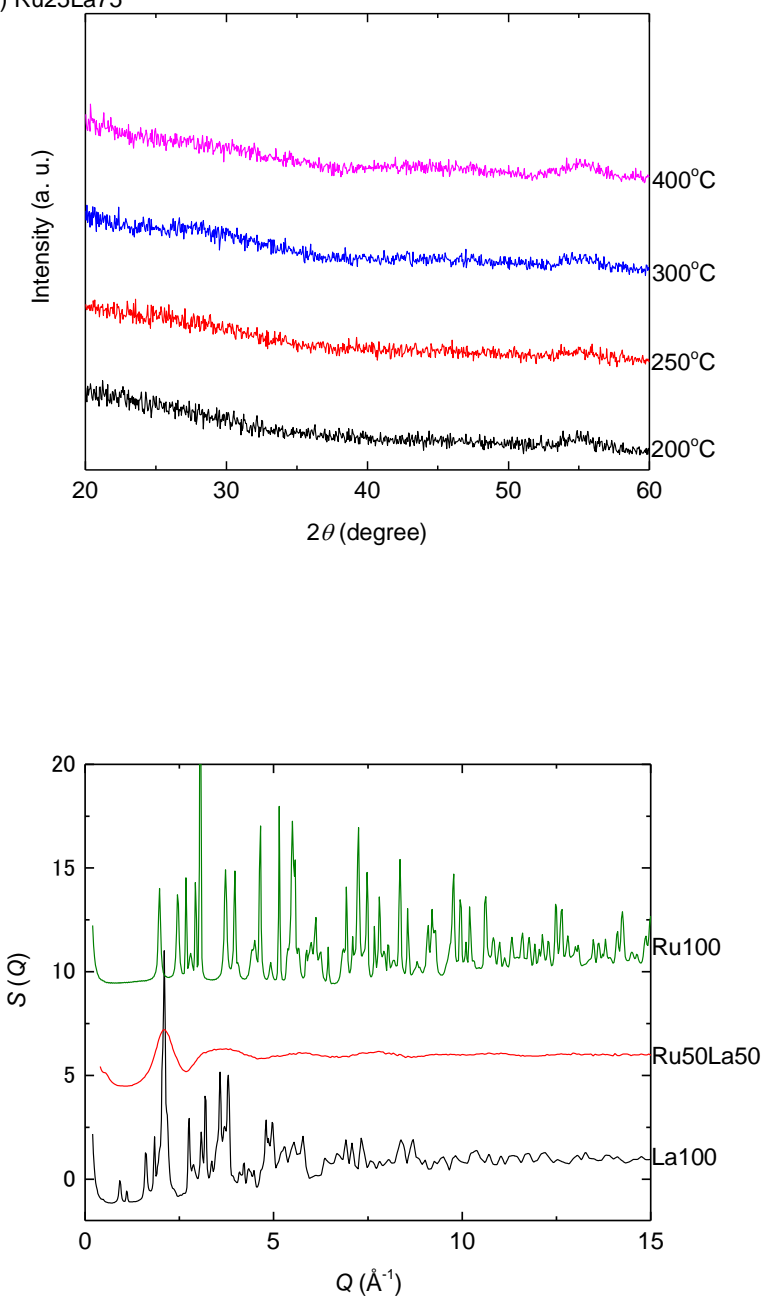
1

2

3

4

5

7

8

9

10

11

12

13

14

15

16

17

18

19

20

21

22

23

24

25

26

27

28

29

30

31

32

33

34

35

36

37

38

39

40

41

42

43

44

45

46

47

48

49

50

51

52

53

54

55

56

57

58

59

60

61

62

63

64

65

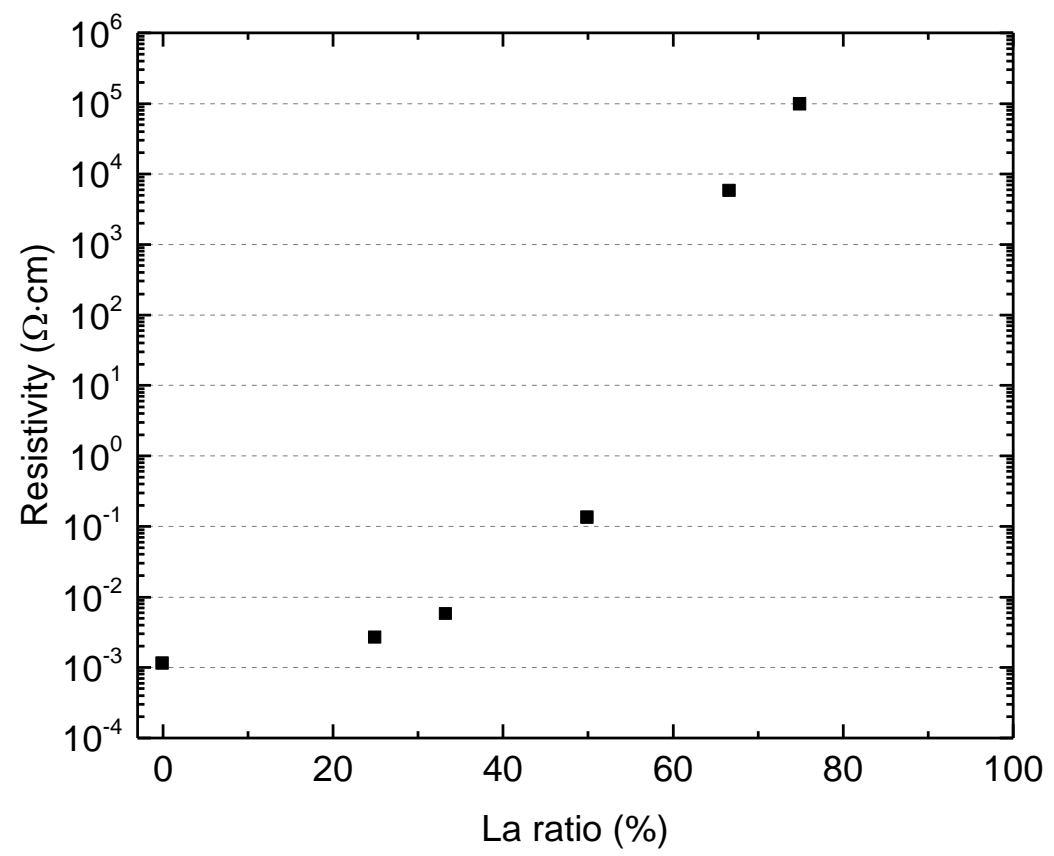

Fig. 6

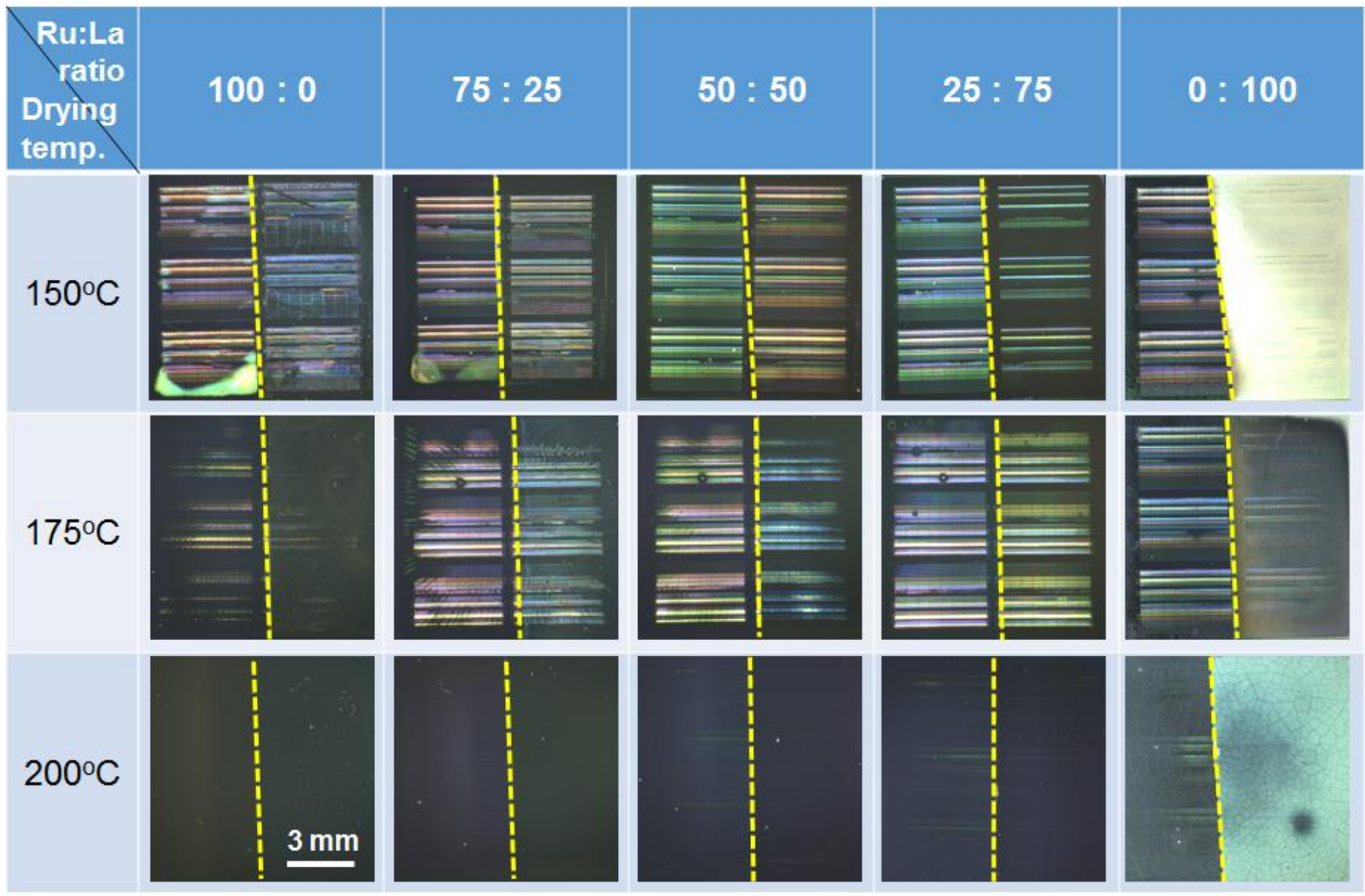

Fig. 7 
(a)

(b)
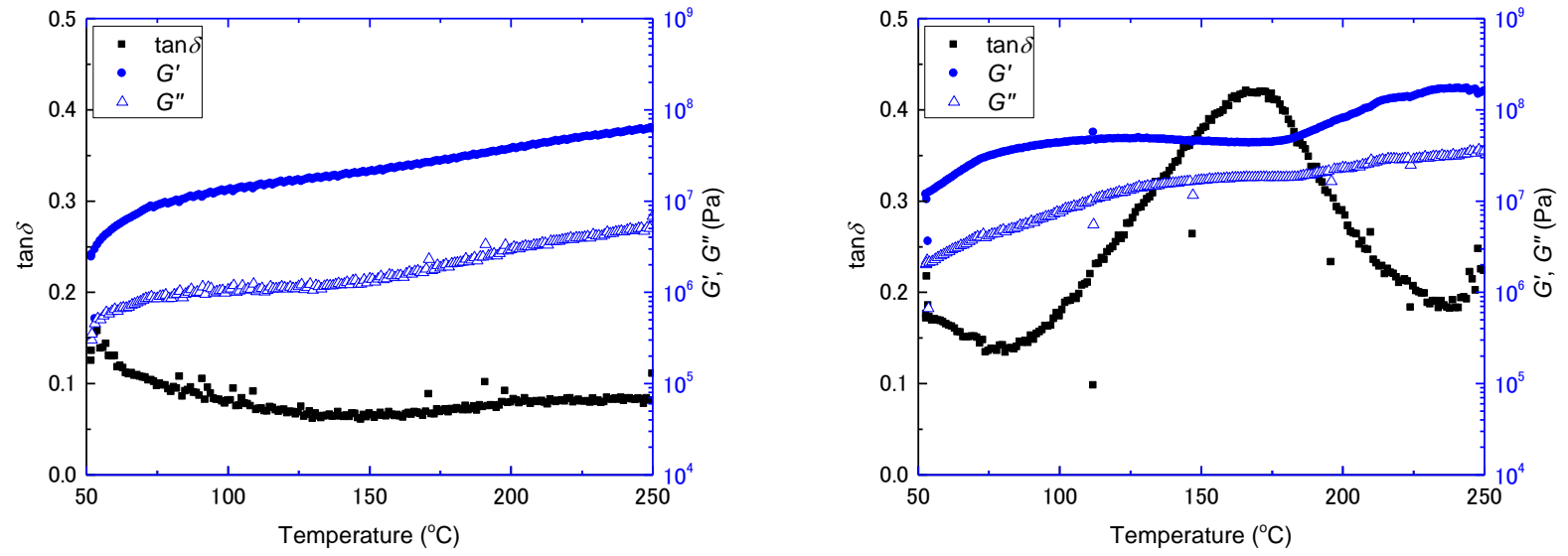

Fig. 8

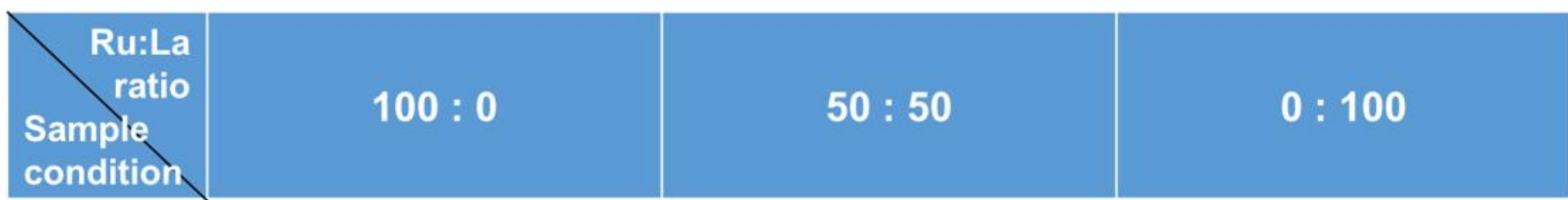

\section{After-} Anneal
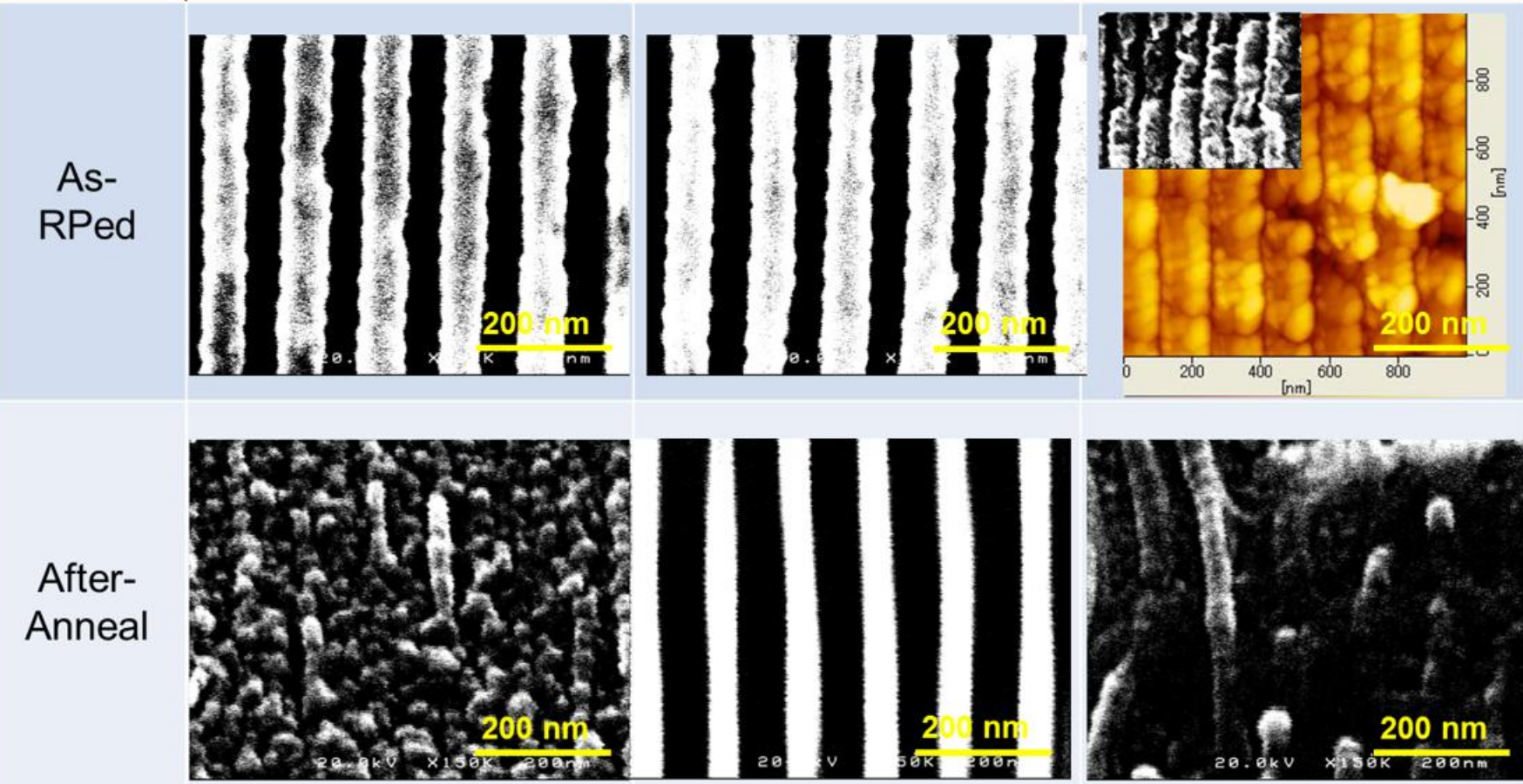

Fig. 9 


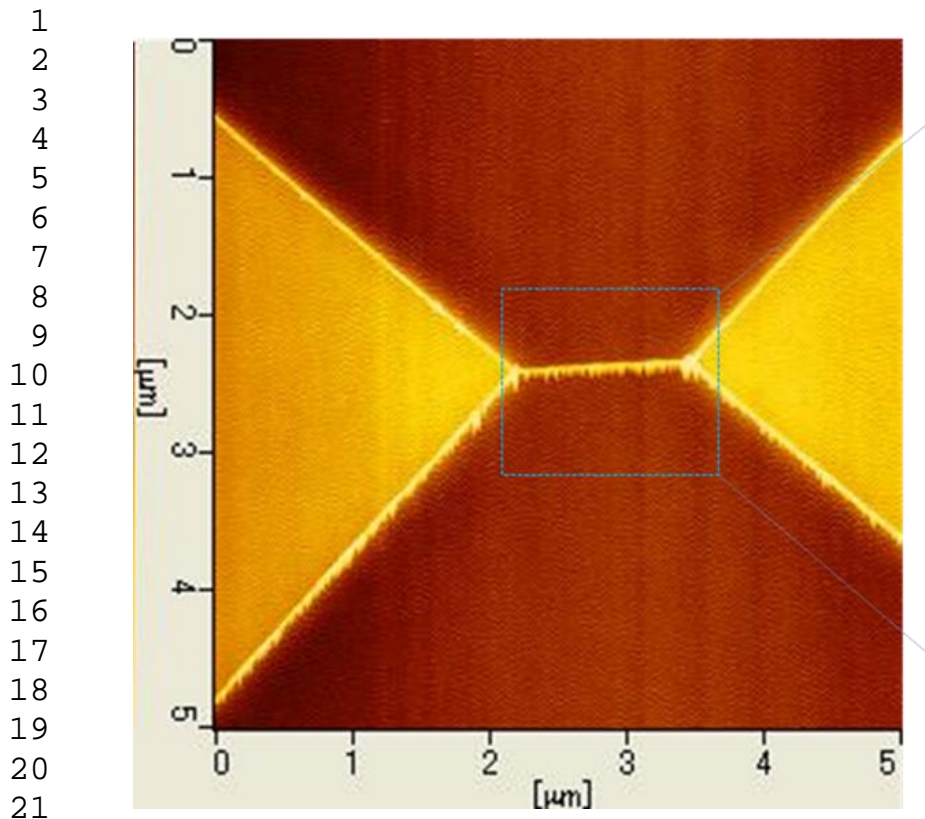

Fig. 10

(a)

(b)

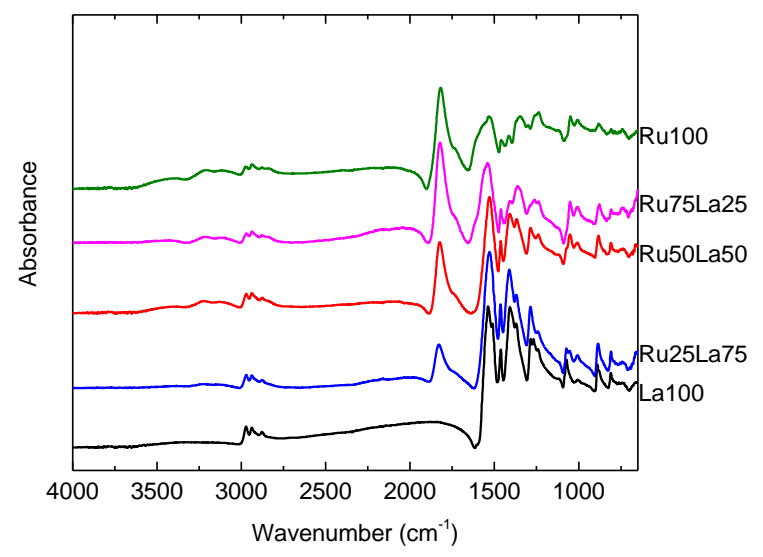

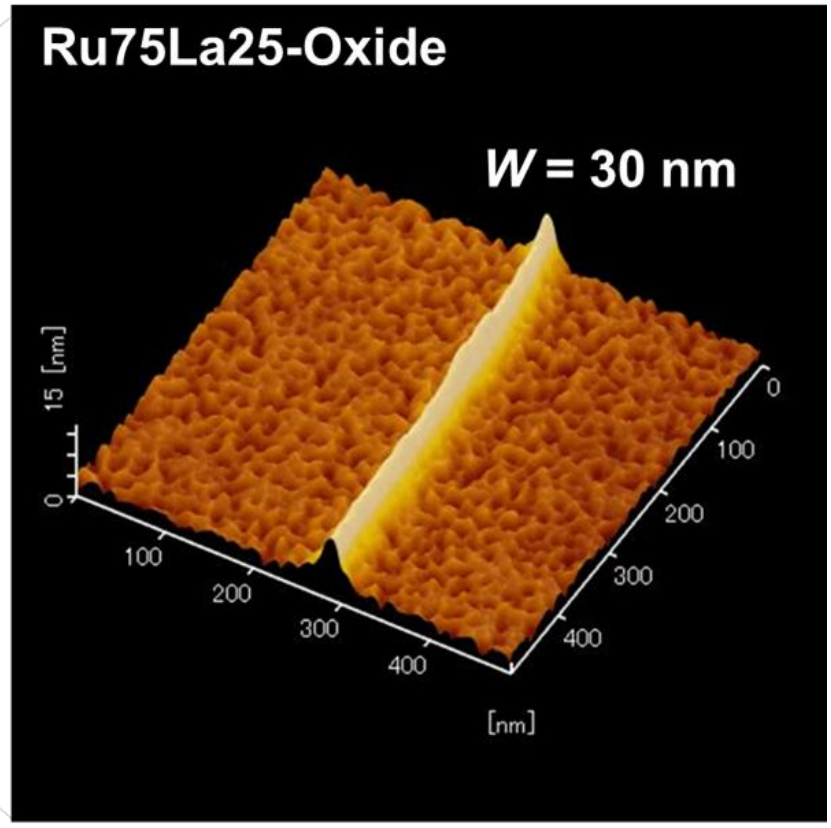

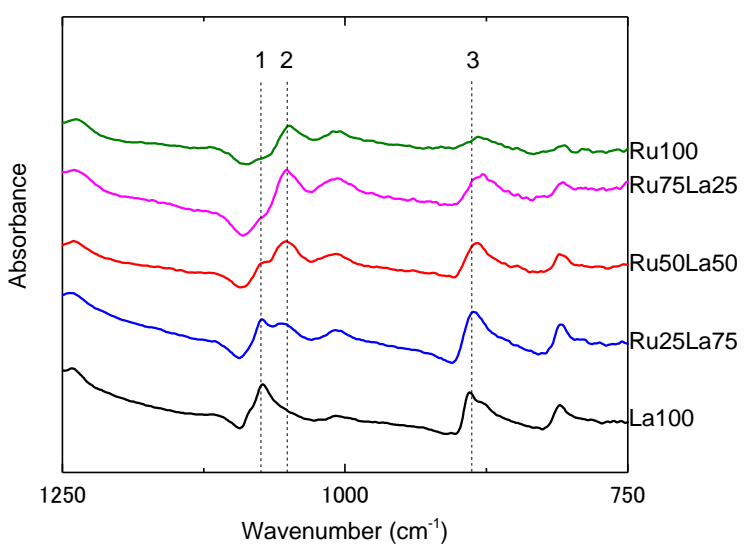

Fig. 11 
1

2

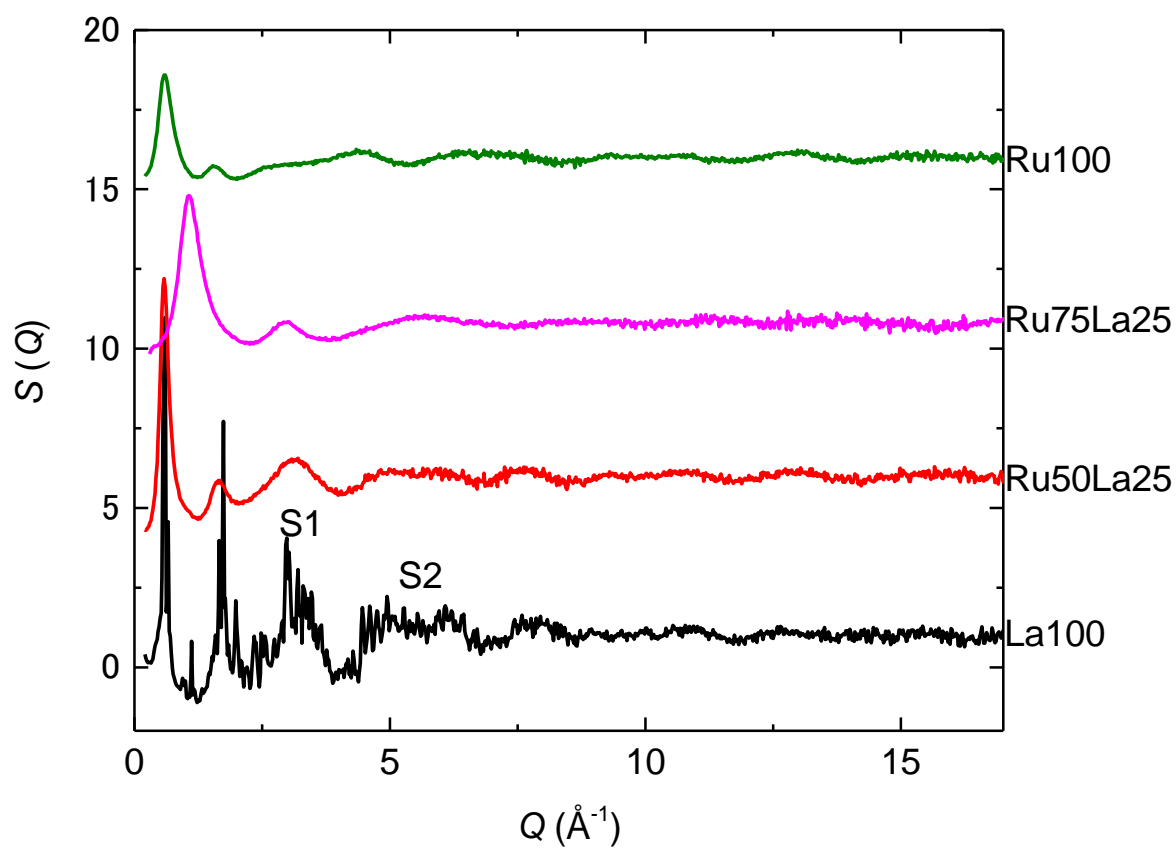

Fig. 12

(a)

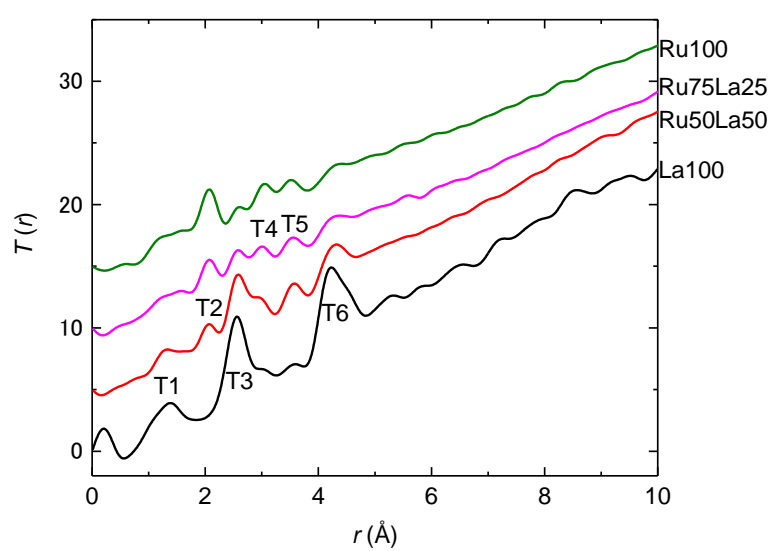

(b)

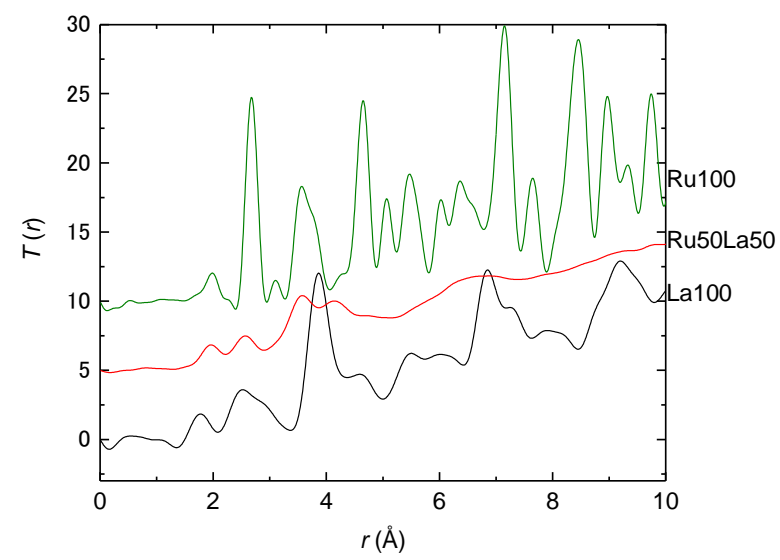

Fig. 13 


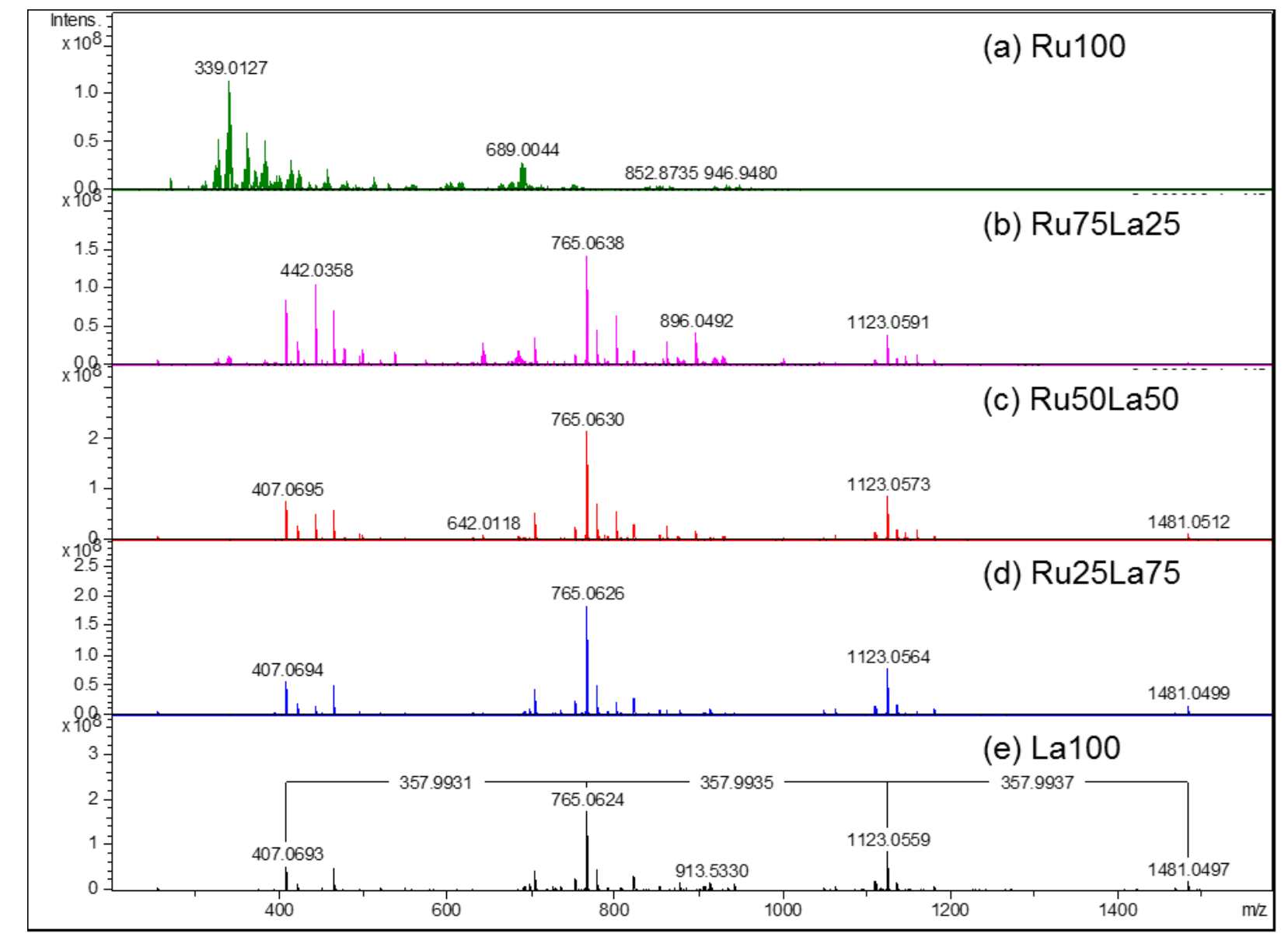

Fig. 14 
Fig. 1 Diagram of the total nano-Rheology Printing (RP) process to form a one-layered pattern.

Fig. 2 Thermogravimetric analysis of RuLa solutions under air at a constant heating rate $\left(10^{\circ} \mathrm{C} \cdot \mathrm{min}^{-1}\right)$. The inset shows an enlarged view from $120-250{ }^{\circ} \mathrm{C}$

Fig. 3 Differential thermal analysis of RuLa solutions under air at a constant heating rate $\left(10^{\circ} \mathrm{C} \cdot \mathrm{min}^{-1}\right)$.

Fig. 4 Differential thermogravimetric analysis of RuLa solutions under air at a constant heating rate $\left(10{ }^{\circ} \mathrm{C} \cdot \mathrm{min}^{-1}\right)$.

Fig. 5 XRD patterns of (a) Ru100, (b) Ru75La25, (c) Ru50La50, (d) Ru25La75 and (e) La100 films prepared under the ambient atmosphere at temperatures ranging from $100{ }^{\circ} \mathrm{C}$ to $400{ }^{\circ} \mathrm{C}$ for $10 \mathrm{~min}$. Panel (e) shows the $\mathrm{S}(\mathrm{Q})$ values of La100-oxide and Ru100-oxide powders annealed at $400{ }^{\circ} \mathrm{C}$, and Ru50La50-oxide annealed at $450{ }^{\circ} \mathrm{C}$, respectively, obtained from HEXRD measurements.

Fig. 6 Room temperature DC resistivity measurements for RuLa-oxide at various RuLa ratios.

Fig. 7 Optical microscope images of the resulting patterns. The left side represents as-RPed patterns,and the right side represents the patterns after annealing at specific ratios of metals and drying temperatures.

Fig. 8 Viscoelastic properties of (a) Ru100-gel and (b) Ru50La50-gel. The $G$ ' and $G$ ' values represent the elastic and viscous moduli, respectively, and $\tan \delta$ is the ratio of $G$ ' to $G$ '.

Fig. 9 SEM and AFM images of as-RPed patterns and annealed patterns (annealed at $400{ }^{\circ} \mathrm{C}$ for 10 min in the ambient atmosphere.)

Fig. 10 AFM images of the Ru75La25-oxide fine pattern with a $30 \mathrm{~nm}$ line width.

Fig. 11 Comparison of the IR spectra obtained from the five RuLa films prepared at $150{ }^{\circ} \mathrm{C}$ for 5 min in the ambient atmosphere at wavenumber ranges from (a) $4000 \mathrm{~cm}^{-1}$ to $650 \mathrm{~cm}^{-1}$, and (b) $1250 \mathrm{~cm}^{-1}$ to $750 \mathrm{~cm}^{-1}$.

Fig. 12 The structure factor $\mathrm{S}(\mathrm{Q})$ of $150{ }^{\circ} \mathrm{C}$-dried RuLa gels measured using HEXRD.

Fig. 13 The total correlation function T(r) of (a) $150{ }^{\circ} \mathrm{C}$-dried RuLa gels and (b) La100-oxide and Ru100-oxide powders annealed at $400^{\circ} \mathrm{C}$, measured using HEXRD.

Fig. 14 CSI-FT-ICR-MS analysis of the five RuLa solutions: (a) Ru100, (b) Ru75La25, (c) Ru50La50, (d) Ru25La75, and (e) La100. 